OPEN ACCESS

Edited by:

Amit K. Dey,

National Institutes of Health (NIH),

United States

Reviewed by:

Shalini Allam,

MedStar Georgetown University Hospital, United States

Stefano Coiro,

Hospital of Santa Maria della

Misericordia in Perugia, Italy

*Correspondence:

Deping Liu

lliudeping@263.net

${ }^{\dagger}$ These authors have contributed equally to this work

Specialty section

This article was submitted to Heart Failure and Transplantation,

a section of the journal

Frontiers in Cardiovascular Medicine

Received: 12 May 2021 Accepted: 17 November 2021 Published: 08 December 2021

Citation:

Xu H, Wang Q, Liu Y, Meng L, Long H,

Wang L and Liu D (2021) U-Shaped Association Between Serum Uric Acid Level and Hypertensive Heart Failure:

A Genetic Matching Case-Control

Study.

Front. Cardiovasc. Med. 8:708581.

doi: 10.3389/fcvm.2021.708581

\section{U-Shaped Association Between Serum Uric Acid Level and Hypertensive Heart Failure: A Genetic Matching Case-Control Study}

\author{
Hongxuan $\mathrm{Xu}^{1,2+}$, Quan Wang ${ }^{3 \dagger}$, Yunqing Liu ${ }^{1,2}$, Lingbing Meng ${ }^{1,4}$, Huanyu Long ${ }^{2}$, \\ Li Wang ${ }^{2,5}$ and Deping Liu ${ }^{1,3,6 *}$
}

${ }^{1}$ Chinese Academy of Medical Sciences, National Center of Gerontology, National Health Commission, Department of Cardiology, Beijing Hospital, Institute of Geriatric Medicine, Beijing, China, ${ }^{2}$ The Key Laboratory of Geriatrics, Chinese Academy of Medical Sciences, Beijing Institute of Geriatrics, Beijing Hospital National Center of Gerontology, Institute of Geriatric Medicine, Beijing, China, ${ }^{3}$ Yuetan Community Health Center, Fuxing Hospital, Capital Medical University, Beijing, China, ${ }^{4}$ Chinese Academy of Medical Science, Peking Union Medical College, Beijing, China, ${ }^{5}$ Chinese Academy of Medical Sciences, Departments of Neurology, National Center of Gerontology, Beijing Hospital, Institute of Geriatric Medicine, Beijing, China, ${ }^{6}$ Peking University Health Science Centre, Peking University Fifth School of Clinical Medicine, Beijing, China

Background: Heart failure (HF) is a global pandemic and lays an added burden on public healthcare. Previous studies indicated that high and low serum uric acid levels are associated with worse outcomes in many diseases. Reduced serum uric acid may not result in a better outcome.

Methods: A comparative, matched cross-sectional study design was implemented. The matching variables were age, sex, BMl, BP, and histories of CKD, CVD, diabetes mellitus, stroke, hyperlipidemia. We reviewed the electronic medical records to identify patients diagnosed with hypertension or hypertensive heart failure (HHF) admitted to Beijing Hospital's cardiology department.

Results: The median age of the two groups after matching are 71 . There are 55.6\% males in the hypertension group and 53.8\% in the heart failure group. Univariate logistic regression analysis showed that UA's quadratic term is significant $(O R=1.00,95 \% \mathrm{Cl}$ 1.00 to 1.00; $P=0.03$ ), which indicated a $u$-shaped relationship between hypertension and HHF. FBS $(O R=0.22,95 \% \mathrm{Cl} 0.05$ to $0.95, p=0.07)$ and $\mathrm{HDL}(O R=1.23,95 \%$ $\mathrm{Cl} 1.00$ to $1.54, P=0.05$ ) were not significant but close.

Conclusion: Our results supported that both low and high uric acid levels were predictive of hypertensive heart failure. Besides, high-density lipoprotein cholesterol and fasting blood sugar were also associated with hypertensive heart failure. Low-density lipoprotein cholesterol was not associated with hypertensive heart failure.

Keywords: uric acid, heart failure, matched, lipids, case-control 


\section{INTRODUCTION}

Heart Failure (HF) is a global pandemic and lays an added burden on public healthcare. It affects $\sim 13.5$ million people aged over 35 years in China and results in 1 million hospitalizations annually in the United States and Europe $(1,2)$. HF is a shared end-stage phase of many cardiac diseases; therefore, HF patients can have mixed etiologies. Hypertension is probably one of the most common risk factors for developing HF. As proof, in the Framingham Heart Study, 91\% of participants' hypertension antedated HF development. Multivariable analyses indicated that hypertension accounts for $39 \%$ of HF cases in men and 59\% in women (3).

Persistent hypertension can lead to HF with normal ejection fraction or HF with reduced ejection fraction, the latter mainly through myocardial infarction (4). Chronically increased stress and neurohormones imposed on the cardiac myocytes caused by high blood pressure (BP) will result in left ventricular hypertrophy, diastolic dysfunction, and eventually hypertensive heart failure (HHF). Thus, HHF is characterized by myocardial abnormality resulting from long-standing arterial hypertension in the absence of any other cardiac disease that can cause LVH or cardiac dysfunction. Progressive extracellular matrix (ECM) accumulation is part of the underlying pathophysiological mechanism for heart failure in patients with pressure overload hypertrophy $(\mathrm{POH})(5,6)$.

Researchers have focused on detecting predictors and hypothesizing the underlying HF pathophysiology. Elevated serum uric acid (SUA) is a marker of inflammatory cytokine activation, insulin resistance, and oxidative stress (7). In addition, hyperuricemia may directly contribute to worsening HF outcomes $(8,9)$. Dyslipidemia, hyperglycemia, and hyperuricemia are widely discussed risk factors or markers of cardiovascular diseases (CVD). The so-called reverse epidemiology of HF has been introduced for years, that is risk factors in the general population, including body mass index (BMI), serum cholesterol, and BP, are positively related to a better outcome in patients with chronic heart failure (CHF) (10).

From this perspective, this study aims to explore the causal relation of uric acid with hypertensive patients developing HHF while adjusting covariates like lipoproteins and blood sugar.

\section{METHODS}

\section{Design and Population}

This single-center, matched, comparative case-control study was conducted at Beijing Hospital. We reviewed the electronic medical records to identify patients diagnosed with hypertension or HHF admitted to Beijing Hospital's cardiology department during Jan 1, 2017, and Dec 31, 2017. Hypertension is defined as $\mathrm{BP} \geq 140 \mathrm{mmHg}$ systolic or $90 \mathrm{mmHg}$ diastolic (11). Due to the universally accepted definition of HHF is unavailable, we set our inclusion criteria for the selection of HHF patients as (a) hypertensive patients; (b) left ventricular alteration by an echocardiographic assessment or Cardiac magnetic resonance imaging; (c) New York Heart Association (NYHA) Classification $\geq$ Class II. The exclusion criteria were patients with (a) chronic respiratory diseases; (b) hyperthyroidism or hypothyroidism; (c) cancer; (d) autoimmune disorders; (e) valvular heart diseases. The actual sample sizes of pre-matching participants were 265 and 264 , respectively.

\section{Data Collection}

We collected demographic characteristics, biochemical tests, and BP from the electronic medical record system. Blood samples were obtained after at least 8 -h fasting the following morning after admission. Demographic characteristics included age; gender; body mass index (BMI); history of diabetes mellitus, hyperlipidemia, cardiovascular diseases (CVD), chronic kidney diseases (CKD), and stroke. Biochemical tests included fasting blood sugar (FBS), triglycerides (TG); total cholesterol (TC); high-density lipoprotein cholesterol (HDL-c); low-density lipoprotein cholesterol (LDL-C); serum uric acid (SUA), and creatinine $(\mathrm{Cr})$.

\section{Genetic Matching}

To control potential confounding covariates and underlying bias, researchers have utilized matching in many fields as a method of causal inference. Propensity score and Mahalanobis distance (MD) matching are currently the most popular methods for matching. However, if the covariates have non-ellipsoidal distributions, MD and a misspecified propensity score model do not perform well $(12,13)$. Genetic matching is a generalization of propensity score and MD, developed by Sekhon and Mebane using an evolutionary search algorithm to maximize the balance of observed covariates across matched treated and control units (14). Genetic Matching matches by minimizing a generalized version of Mahalanobis distance (GMD) that is:

$$
G M D\left(X_{i}, X_{j}, W\right)=\sqrt{\left(X_{i}-X_{j}\right)^{T}\left(S^{-\frac{1}{2}}\right)^{T} W S^{-\frac{1}{2}}\left(X_{i}-X_{j}\right)}
$$

$\mathrm{W}$ is a $\mathrm{k} \times \mathrm{k}$ positive definite weight matrix, and $S^{-\frac{1}{2}}$ is the Cholesky decomposition of S.

The matching variables were age, sex, BMI, BP, and histories of CKD, CVD, diabetes mellitus, stroke, hyperlipidemia.

\section{Statistical Analysis}

All data were analyzed using $\mathrm{R}$ 4.0.5. The major packages used for matching were "matching," "cobalt," and "matchit." Comparisons between 2 groups of continuous variables were assessed for statistical significance by unpaired Student $t$-test or $z$-test, and binary variables by Pearson's $\chi^{2}$ test. Potential risk factors were first analyzed by univariable logistic regression analysis. Statistically significant predictors were further analyzed for their independent predictive value using multivariable logistic modeling. We used the Hosmer-Lemeshow tests to test the goodness of fit of logistic regression models. Likelihood ratio $\left(\mathrm{LR} \chi^{2}\right)$ tests were used to compare the fitness of two statistical models. The significance level was defined as $P<0.05$. All the hypothesis tests were 2 -sided. 
TABLE 1 | Demographic characteristics.

\begin{tabular}{|c|c|c|c|c|c|c|}
\hline \multirow[b]{2}{*}{ Clinical Values } & \multicolumn{2}{|c|}{ Before matching } & \multirow[b]{2}{*}{$P$-value } & \multicolumn{2}{|c|}{ After matching } & \multirow[b]{2}{*}{$P$-value } \\
\hline & $\begin{array}{l}\text { Hypertension } \\
\text { (N=265) }\end{array}$ & $\begin{array}{c}\text { HHF } \\
(N=264)\end{array}$ & & $\begin{array}{l}\text { Hypertension } \\
\text { (N=72) }\end{array}$ & $\begin{array}{c}\text { HHF } \\
(N=65)\end{array}$ & \\
\hline Age, median (IQR) & $66[26,96]$ & $72[37,91]$ & $<0.001$ & $71[53,91]$ & $71[37,88]$ & 0.88 \\
\hline Male No. (\%) & 135 (51\%) & $147(56 \%)$ & 0.315 & $40(56 \%)$ & 35 (54\%) & 0.977 \\
\hline BMl & $26(16,38)$ & $25(17,37)$ & 0.284 & $25.7(4)$ & $25.8(4)$ & 0.897 \\
\hline \multicolumn{7}{|c|}{ Blood pressure, mean (SD), mmHg } \\
\hline Systolic & $143(21)$ & $141(20)$ & 0.38 & $145(18)$ & $144(21)$ & 0.968 \\
\hline Diastolic & $82(14)$ & $76(12)$ & $<0.001$ & $78(11)$ & $78(11)$ & 0.964 \\
\hline Diabetes No. (\%) & $80(30 \%)$ & $125(47 \%)$ & $<0.001$ & $25(35 \%)$ & $25(39 \%)$ & 0.782 \\
\hline CVD No. (\%) & 47 (18\%) & $233(88 \%)$ & $<0.001$ & $45(63 \%)$ & $44(68 \%)$ & 0.648 \\
\hline Hyperlipidemia No. (\%) & 231 (87\%) & 254 (96\%) & $<0.001$ & 65 (90\%) & $60(92 \%)$ & 0.907 \\
\hline Stroke No. (\%) & 37 (14\%) & 54 (21\%) & 0.062 & $9(13 \%)$ & $9(14 \%)$ & $>0.99$ \\
\hline CKD No. (\%) & $19(7 \%)$ & 39 (15\%) & 0.008 & $10(14 \%)$ & $10(15 \%)$ & $>0.99$ \\
\hline
\end{tabular}

BMI, body mass index; CVD, cardiovascular diseases; CKD, chronic kidney diseases.

\section{RESULTS}

\section{Characteristics and Genetic Matching}

A total of 529 individuals were selected from all 2,722 records, 265 were included in the hypertension group and 264 in the HHF group to perform genetic matching. After 1:1 matching twice, 137 individuals were successfully matched. Table 1 showed the essential demographic characteristics and medical histories before and after matching. The difference of all covariates between the two groups after matching reached insignificance, indicating that genetic matching yields an equilibrious distribution of covariates, leading to a comparable paired dataset. Figure 1 presented two Love-plots (left was the first matching and right the second) with the balance statistic on the $\mathrm{X}$-axis of each matching, all the points after adjustment were within the 0.1 standardized mean difference threshold. It also was good evidence that balance had been achieved. We exchanged the data between both groups to perform the second matching and exchanged it back after matching, so the red points of the right plot are vertically flipped regarding the green points of the left. Table 2 summarized the potential risk factors examined in this study.

\section{Logistic Regression}

Univariate logistic regression analysis (Table 3 ) showed that UA's quadratic term is significant $(O R=1.00,95 \% \mathrm{CI}-1.00$ to $1.00 ; P=0.03$ ), which indicated a u-shaped relationship between hypertension and HHF. FBS (OR $=0.22,95 \% \mathrm{CI}$ 0.05 to $0.95, p=0.07)$ and HDL $(O R=1.23,95 \%$ CI 1.00 to $1.54, P=0.05)$ were statistically not significant but close. The confidence intervals of the odds ratio (OR) of both were either above or below 1. For 1 unit increased in FBS, the odds of developing HHF increased by a factor of 1.23. For 1 unit increased in HDL-c, the odds of developing HHF were 4.55 times lesser. Figure 2 presented the relationships between UA and the probability of developing HHF. The probability of HHF regarding SUA was lowest at around $200-300 \mu \mathrm{mol} / \mathrm{L}$, lower or higher uric acid concentrations were both positively associated with the incidence of HHF.

The multivariate logistic regression analysis results, including FBS, HDL, and UA, were similar to the univariate logistic regression analysis Table 3. The Hosmer-Lemeshow $\left(\chi^{2}=6.48\right.$, $d f=8, p=0.59)$ tests indicated evidence of a good fit model. Moreover, the likelihood ratio test compared the multivariate logistic regression model with the model without predictors yielded a significant result $\left(\operatorname{LR} \chi^{2}=14.93, d f=4, p=0.005\right)$.

We then tested the interactions among variables. The interaction between FBS and UA in the multivariate logistic regression analysis was not significant $(O R=0.99, p=0.08)$. The likelihood ratio test compared the multivariate model with the model without interaction yielded a not significant result $(p=0.07)$.

\section{DISCUSSION}

In the present study, we examined the association with multiple variates to patients with HHF. The results demonstrated that low and high serum UA levels were significantly associated with the probability of developing HHF, videlicet the U-shaped association between serum UA levels and HHF. On the other hand, HDL was negatively associated with HHF.

\section{Serum Uric Acid}

Uric acid is correlated with blood pressure in children with new-onset, untreated, primary hypertension, $90 \%$ of newly diagnosed adolescent hypertensives are hyperuricemic (15). Urate lowering therapies may mitigate hypertension caused by hyperuricemia (16).

It is still controversial what role uric acid (UA) plays in heart failure. Conflicting current studies' findings suggest a reciprocal causality of the relationship between SUA elevation and CVD (17-20). Lowering uric acid alone may not improve the prognosis of patients with heart failure (21). In large cohort studies of the general population, associations between the 


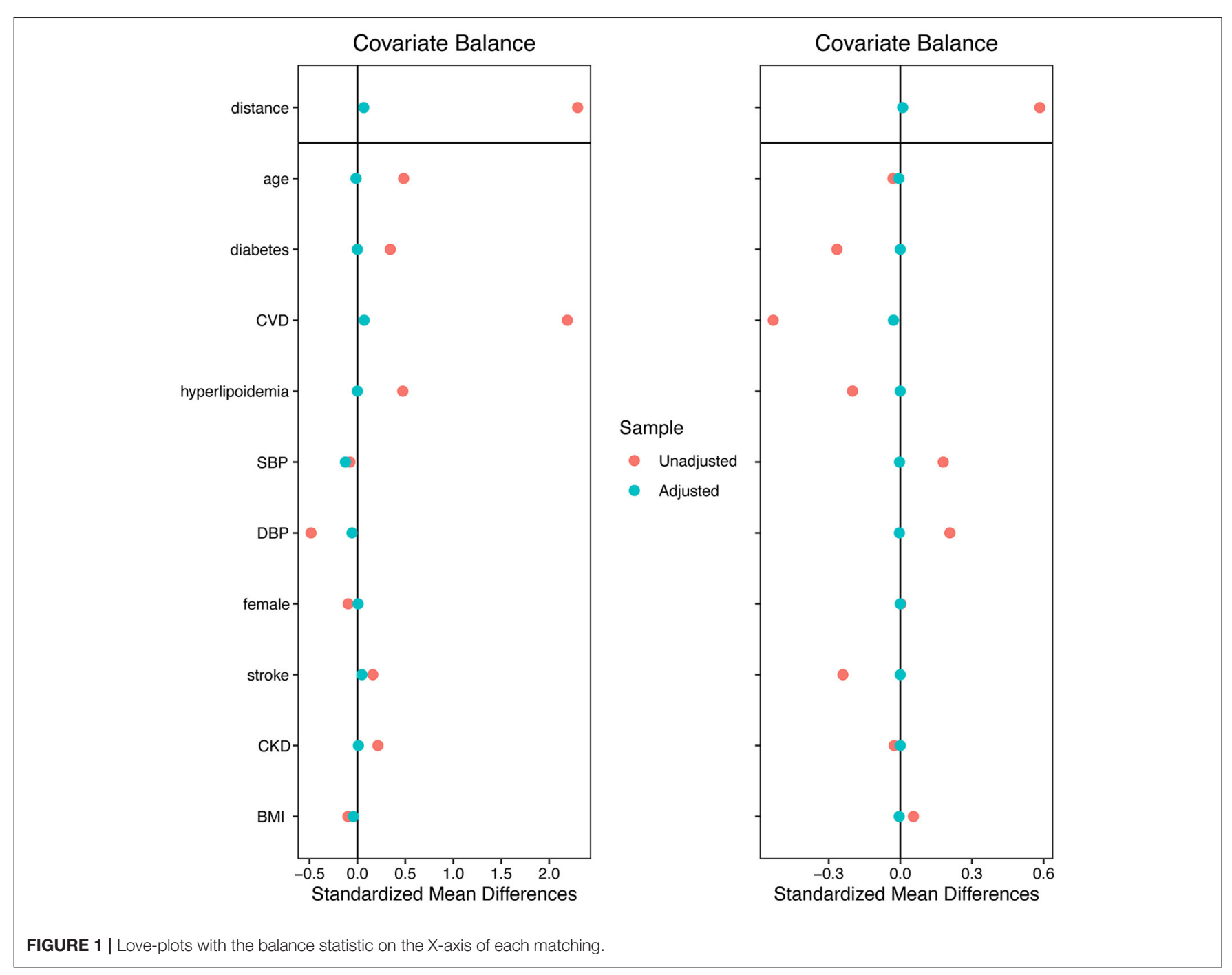

TABLE 2 | The potential risk factors.

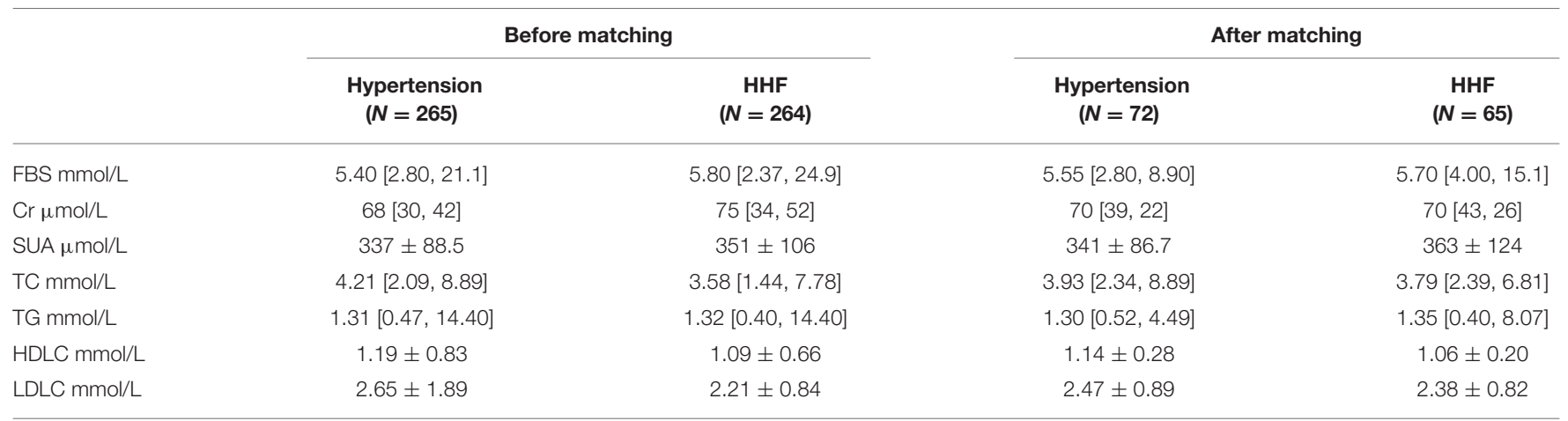

FBS, fasting blood sugar; TG, triglycerides; TC, total cholesterol; HDLC, high-density lipoprotein cholesterol; LDLC, low-density lipoprotein cholesterol; SUA, serum uric acid; $\mathrm{Cr}$, creatinine.

whole range levels of SUA and prognosis supported a U-shaped association $(22,23)$. Epidemiological studies have suggested that hyperuricemia is a clinically significant risk factor or marker of lifestyle-related diseases that are the harbingers of HF such as hypertension, $\mathrm{CKD}$, cerebrocardiovascular disease, metabolic syndrome (24-27). Hypoxemia due to HF may increase xanthine oxidase activity in cardiomyocytes and endothelial cells, resulting in increased SUA levels. Furthermore, Diuretic drugs are commonly used to relieve clinical HF symptoms, and they can induce hyperuricemia through increasing UA reabsorption and 
TABLE 3 | Logistic regression results.

\begin{tabular}{|c|c|c|c|c|c|}
\hline & B & SE & WALD & $P$-value & OR $(95 \% \mathrm{Cl})$ \\
\hline \multicolumn{6}{|c|}{ Univariate Logistic Regression } \\
\hline TC & -0.1444 & 0.1735 & 0.692 & 0.405 & $0.87[0.61,1.21]$ \\
\hline TG & 0.2557 & 0.2062 & 1.540 & 0.210 & $1.29[0.88,2.01]$ \\
\hline LDL-C & -0.1279 & 0.2063 & 0.384 & 0.535 & $0.88[0.58,1.31]$ \\
\hline HDL-C & -1.4963 & 0.7727 & 3.748 & 0.053 & $0.22[0.05,0.95]$ \\
\hline FBS & 0.2030 & 0.1102 & 3.397 & 0.065 & $1.23[1.00,1,54]$ \\
\hline $\mathrm{Cr}$ & 0.006810 & 0.005055 & 1.814 & 0.178 & $1.01[1.00,1.02]$ \\
\hline UA & $-2.044 \mathrm{E}-02$ & $1.055 \mathrm{E}-02$ & 3.752 & 0.053 & $0.98[0.96,1.00]$ \\
\hline $\begin{array}{l}\cup A^{*} \\
\text { UA }\end{array}$ & 3.098E-05 & 1.453E-05 & 4.545 & 0.033 & $1.00[1.00,1.00]$ \\
\hline \multicolumn{6}{|c|}{ Multivariate Logistic Regression } \\
\hline UA & $-2.204 \mathrm{E}-02$ & 1.127E-02 & 3.830 & 0.0504 & $0.98[0.95,1.00]$ \\
\hline $\begin{array}{l}\cup A^{*} \\
\text { UA }\end{array}$ & 3.232E-05 & $1.525 \mathrm{E}-05$ & 4.490 & 0.0341 & $1.00[1.00,1.00]$ \\
\hline HDL-C & 1.983E-01 & $1.129 \mathrm{E}-01$ & 3.087 & 0.0790 & $0.24[0.04,1.09]$ \\
\hline FBS & $-1.437 \mathrm{E}+00$ & 8.091E-01 & 3.154 & 0.0757 & $1.22[0.99,1.55]$ \\
\hline
\end{tabular}

FBS, fasting blood sugar; TG, triglycerides; TC, total cholesterol; HDLC, high-density lipoprotein cholesterol; LDLC, low-density lipoprotein cholesterol; SUA, serum uric acid; $\mathrm{Cr}$, creatinine.

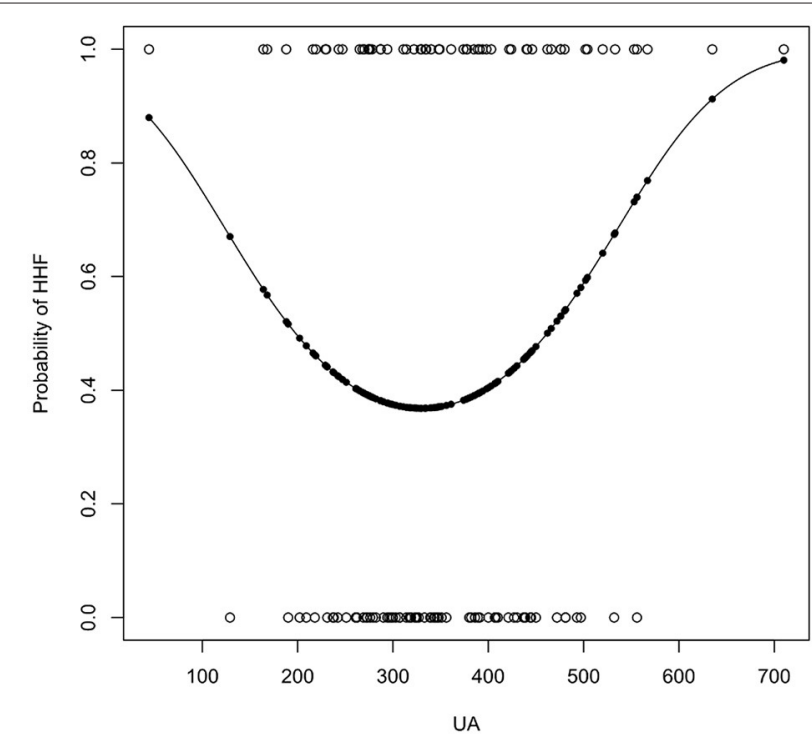

FIGURE 2 | The result of univariate logistic regression of UA. The $x$-axis is the concentration of uric acid $(\mu \mathrm{mol} / \mathrm{L})$.

decreasing UA secretion. It is intuitive to suspect a relationship between UA and HF, and hyperuricemia is indeed related to worse prognosis in HF patients (28).

Patients with hypouricemia were characterized by older age, women, low BMI, non-smokers, statins, and diabetes. Patients with hyperuricemia were characterized by male sex, dyslipidemia, dilated cardiomyopathy, atrial fibrillation, history of HF admission, use of diuretics, low LVEF, and sizeable left ventricle (28). We performed genetic matching to equilibrate these covariates to output a more reliable analysis.
High SUA was found to be an independent prognosticator of adverse outcomes of HF outpatients in cohort studies $(8,9)$. PARAGON-HF showed that sacubitril-valsartan treated HFpEF patients had the lowest incidence rates of the primary composite endpoint, cardiovascular death, heart failure hospitalization, allcause death, myocardial infarction, and stroke, with baseline SUA levels around $4-6 \mathrm{mg} / \mathrm{dl}(238-357 \mu \mathrm{mol} / \mathrm{L})$ (29). Our results indicated that SUA has a U-shaped relationship with the HHF with a similar SUA concentration. The mechanisms of the relation between low UA levels and HHF remain to be elucidated.

\section{Lipoproteins and Blood Sugar}

Freeman et al. showed that the deposition of cholesterolrich microdomains in the macrophage's surface and the ECM resulting from acetylated (Ac)-LDL loading could be inhibited by the presence of ApoA-I (the major component of HDL particles) in vitro (30). Low-density lipoprotein cholesterol (LDL-c), highdensity lipoprotein cholesterol (HDL-c), and triglycerides were regarded as predictors of outcomes in HF patients (31-33). Yet, a recent study showed that LDL-c was not associated with cardiovascular outcomes in overweight or obese subjects (34). We also did not detect a significant difference of LDL-c between hypertension and HHF.

Hyperglycemia is a conventional status in HF patients. Heart failure is associated with systemic insulin resistance due to insulin resistance, lack of movement, or exercise. Insulin resistance may lead to HDL dysfunction (35). HF patients with glucose metabolism alterations are more likely to be hospitalized than patients free from diabetes mellitus (36).

$\mathrm{HDL}$ is envisioned as a scavenger molecule that can reverse cholesterol transport and has anti-inflammatory, anti-oxidative, immunomodulatory, and endothelial protective properties. These properties are highly dependent on the proteome and lipidome of the particles (such as apolipoprotein AI) and do not necessarily correlate with HDL-c levels. Low HDL or ApoA-I levels independently correlate with worsening prognosis in advanced HF $(34,37)$. Apolipoprotein $M$ (apoM) is a multifunctional lipid mediator on HDL that was observed to be negatively correlated with BMI and the insulin resistance index, suggesting that apoM exerts protective roles against the development of insulin resistance (38). Studies in vivo with established heart failure have demonstrated that recombinant $\mathrm{HDL}_{\mathrm{Milano}}$ induces regression of interstitial fibrosis and normalizes lung weight in mice with established heart failure (39). Taken together, HDL appears to play a substantial role in the entire progression of HHF. The addition of FBS and HDL-c to the model made our results of SUA-HHF correlation more reliable.

\section{Limitations}

Several limitations of our study should be mentioned. First, the sample size after matching is relatively small, so the results should be carefully interpreted, for a small sample size has more chances to yield false-negative results. Secondly, we did not include arrhythmia and NYHA I patients in the matching, and atrial fibrillation is the most frequent cardiac complication in hypertensive patients. Lastly, we did not evaluate and stratify the patients based on the ejaculate fraction. These can lead 
to potential underlying bias and need caution generalizing the present findings.

\section{CONCLUSION}

Our results supported a U-shaped causal association between serum uric acid levels and hypertensive heart failure, which means both low and high uric acid levels were predictive of hypertensive heart failure.

\section{DATA AVAILABILITY STATEMENT}

The original contributions presented in the study are included in the article/Supplementary Material, further inquiries can be directed to the corresponding author.

\section{ETHICS STATEMENT}

The studies involving human participants were reviewed and approved by Ethics Committee of the Beijing Hospital (No. 2021BJYYEC-160-02). Written informed consent for participation was not required for this study in accordance with the national legislation and the institutional requirements.

\section{REFERENCES}

1. Hao G, Wang X, Chen Z, Zhang L, Zhang Y, Wei B, et al. Prevalence of heart failure and left ventricular dysfunction in China: the China Hypertension Survey, 2012-2015. Eur J Heart Fail. (2019) 21:1329-37. doi: 10.1002/ejhf.1629

2. Ambrosy AP, Fonarow GC, Butler J, Chioncel O, Greene SJ, Vaduganathan $\mathrm{M}$, et al. The global health and economic burden of hospitalizations for heart failure: lessons learned from hospitalized heart failure registries. J Am Coll Cardiol. (2014) 63:1123-33. doi: 10.1016/j.jacc.2013.11.053

3. Levy D, Larson MG, Vasan RS, Kannel WB, Ho KKL. The progression from hypertension to congestive heart failure. JAMA. (1996) 275:155762. doi: 10.1001/jama.1996.03530440037034

4. Richards AM, Nicholls MG, Troughton RW, Lainchbury JG, Elliot J, Frampton C, et al. Antecedent hypertension and heart failure after myocardial infarction. J Am Coll Cardiol. (2002) 39:1182-8. doi: 10.1016/s0735-1097(02)01737-0

5. Katz AM, Zile MR. New molecular mechanism in diastolic heart failure. Circulation. (2006) 113:19225. doi: 10.1161/CIRCULATIONAHA.106.620765

6. Zile MR, Baicu CF, Gaasch WH. Diastolic heart failure-abnormalities in active relaxation and passive stiffness of the left ventricle. N Engl J Med. (2004) 350:1953-9. doi: 10.1056/NEJMoa032566

7. Borghi C, Palazzuoli A, Landolfo M, Cosentino E. Hyperuricemia: a novel old disorder-relationship and potential mechanisms in heart failure. Heart Fail Rev. (2020) 25:43-51. doi: 10.1007/s10741-019-09869-z

8. Coiro S, Carluccio E, Biagioli P, Alunni G, Murrone A, D'Antonio A, et al. Elevated serum uric acid concentration at discharge confers additive prognostic value in elderly patients with acute heart failure. Nutr Metab Cardiovasc Dis. (2018) 28:361-8. doi: 10.1016/j.numecd.2017.12.009

9. Ambrosio G, Leiro MGC, Lund LH, Coiro S, Cardona A, Filippatos G, et al. Serum uric acid and outcomes in patients with chronic heart failure through the whole spectrum of ejection fraction phenotypes: analysis of the ESCEORP Heart Failure Long-Term (HF LT) registry. Eur J Intern Med. (2021) 89:65-75. doi: 10.1016/j.ejim.2021.04.001

10. Kalantar-Zadeh K, Block G, Horwich T, Fonarow GC. Reverse epidemiology of conventional cardiovascular risk factors in patients with chronic heart failure. J Am Coll Cardiol. (2004) 43:1439-44. doi: 10.1016/j.jacc.2003.11.039

\section{AUTHOR CONTRIBUTIONS}

HX was the major contributor to writing. YL and QW were involved in data collecting. DL made substantial contributions to research conception and designed the draft of the research process. HL gives technical support in the statistical methods. All authors read and approved the final manuscript.

\section{FUNDING}

The Chinese Academy of Medical Sciences funded the present study, CAMS Innovation Fund for Medical Sciences (Grant No. 2018-I2M-1-002), National Key R\&D Program of China (Grant No. 2020YFC2003000), and National Natural Science Foundation of China (Grant Nos. 31271097 and 51672030).

\section{SUPPLEMENTARY MATERIAL}

The Supplementary Material for this article can be found online at: https://www.frontiersin.org/articles/10.3389/fcvm. 2021.708581/full\#supplementary-material

11. Chobanian AV, Bakris GL, Black HR, Cushman WC, Green LA, Izzo JL Jr, et al. Seventh report of the joint national committee on prevention, detection, evaluation, and treatment of high blood pressure. Hypertension. (2003) 42:1206-52. doi: 10.1161/01.HYP.0000107251.49515.c2

12. Rubin DB. Multivariate matching methods that are equal percent bias reducing, II: maximums on bias reduction for fixed sample sizes. ETS Res Bull Ser. (1974) 1974:i-26. doi: 10.1002/j.2333-8504.1974.tb00866.x

13. Rubin DB, Thomas N. Affinely invariant matching methods with ellipsoidal distributions. Ann Stat. (1992) 20:1079-93. doi: 10.1214/aos/1176348671

14. Sekhon JS, Mebane WR. Genetic optimization using derivatives. Polit Anal. (1998) 7:187-210. doi: 10.1093/pan/7.1.187

15. Feig DI, Johnson RJ. Hyperuricemia in childhood primary hypertension. Hypertension. (2003) 42:247-52. doi: 10.1161/01.HYP.0000085858.66548.59

16. Soletsky B, Feig DI. Uric acid reduction rectifies prehypertension in obese adolescents. Hypertension. (2012) 60:114856. doi: 10.1161/HYPERTENSIONAHA.112.196980

17. Huang G, Qin J, Deng X, Luo G, Yu D, Zhang M, et al. Prognostic value of serum uric acid in patients with acute heart failure: a meta-analysis. Medicine (Baltimore). (2019) 98:e14525. doi: 10.1097/MD.0000000000014525

18. Breuer GS, Schwartz Y, Freier-Dror Y, Nesher G. Uric acid level as predictor of mortality in the acute care setting of advanced age population. Eur J Intern Med. (2017) 44:74-6. doi: 10.1016/j.ejim.2017.07.021

19. Bredemeier M, Lopes LM, Eisenreich MA, Hickmann S, Bongiorno GK. d'Avila R, Morsch ALB, da Silva Stein F, Campos GGD. Xanthine oxidase inhibitors for prevention of cardiovascular events: a systematic review and meta-analysis of randomized controlled trials. BMC Cardiovasc Disord. (2018) 18:24. doi: 10.1186/s12872-018-0757-9

20. Carluccio E, Coiro S, Ambrosio G. Unraveling the relationship between serum uric acid levels and cardiovascular risk. Int J Cardiol. (2018) 253:174175. doi: 10.1016/j.ijcard.2017.11.035

21. Xu H, Liu Y, Meng L, Wang L, Liu D. Effect of uric acid-lowering agents on patients with heart failure: a systematic review and metaanalysis of randomised controlled trials. Front Cardiovasc Med. (2021) 8:639392. doi: 10.3389/fcvm.2021.639392

22. Cho SK, Chang Y, Kim I, Ryu S. U-shaped association between serum uric acid level and risk of mortality: a cohort study. Arthritis Rheumatol. (2018) 70:1122-32. doi: 10.1002/art.40472 
23. Hu L, Hu G, Xu BP, Zhu L, Zhou W, Wang T, Bao H, Cheng X. Ushaped association of serum uric acid with all-cause and cause-specific mortality in US adults: a cohort study. J Clin Endocrinol Metab. (2020) 105:dgz068. doi: 10.1210/clinem/dgz068

24. Grayson PC, Kim SY, LaValley M, Choi HK. Hyperuricemia and incident hypertension: a systematic review and meta-analysis. Arthritis Care Res (Hoboken). (2011) 63:102-10. doi: 10.1002/acr.20344

25. Zhu P, Liu Y, Han L, Xu G, Ran JM. Serum uric acid is associated with incident chronic kidney disease in middle-aged populations: a meta-analysis of 15 cohort studies. PLoS ONE. (2014) 9:e100801. doi: 10.1371/journal.pone.0100801

26. Li M, Hu X, Fan Y, Li K, Zhang X, Hou W, et al. Hyperuricemia and the risk for coronary heart disease morbidity and mortality a systematic review and dose-response meta-analysis. Sci Rep. (2016) 6:19520. doi: 10.1038/srep 19520

27. Yuan H, Yu C, Li X, Sun L, Zhu X, Zhao C, et al. Serum uric acid levels and risk of metabolic syndrome: a dose-response meta-analysis of prospective studies. J Clin Endocrinol Metab. (2015) 100:4198-207. doi: 10.1210/jc.2015-2527

28. Mantovani A, Targher G, Temporelli PL, Lucci D, Gonzini L, Nicolosi GL, et al. Prognostic impact of elevated serum uric acid levels on longterm outcomes in patients with chronic heart failure: A post-hoc analysis of the GISSI-HF (Gruppo Italiano per lo Studio della Sopravvivenza nella Insufficienza Cardiaca-Heart Failure) trial. Metabolism. (2018) 83:20515. doi: 10.1016/j.metabol.2018.02.007

29. Selvaraj S, Claggett BL, Pfeffer MA, Desai AS, Mc Causland FR, McGrath MM, et al. Serum uric acid, influence of sacubitril-valsartan, and cardiovascular outcomes in heart failure with preserved ejection fraction: PARAGON-HF. Eur J Heart Fail. (2020) 22:2093-101. doi: 10.1002/ejhf.1984

30. Freeman SR, Jin X, Anzinger JJ, Xu Q, Purushothaman S, Fessler MB, et al. ABCG1-mediated generation of extracellular cholesterol microdomains. $J$ Lipid Res. (2014) 55:115-27. doi: 10.1194/jlr.M044552

31. Charach G, George J, Roth A, Rogowski O, Wexler D, Sheps D, et al. Baseline low-density lipoprotein cholesterol levels and outcome in patients with heart failure. Am J Cardiol. (2010) 105:100-4. doi: 10.1016/j.amjcard.2009.08.660

32. Mehra MR, Uber PA, Lavie CJ, Milani RV, Park MH, Ventura HO. Highdensity lipoprotein cholesterol levels and prognosis in advanced heart failure. J Heart Lung Transplant. (2009) 28:876-80. doi: 10.1016/j.healun.2009.04.026

33. Schaefer EJ, Anthanont P, Diffenderfer MR, Polisecki E, Asztalos BF. Diagnosis and treatment of high density lipoprotein deficiency. Prog Cardiovasc Dis. (2016) 59:97-106. doi: 10.1016/j.pcad.2016.08.006
34. Castañer O, Pintó X, Subirana I, Amor AJ, Ros E, Hernáez Á, et al. Remnant cholesterol, not LDL cholesterol, is associated with incident cardiovascular disease. J Am Coll Cardiol. (2020) 76:2712-24. doi: 10.1016/j.jacc.2020.10.008

35. Ceia F, Fonseca C, Mota T, Morais H, Matias F, de Sousa A, et al. Prevalence of chronic heart failure in Southwestern Europe: the EPICA study. Eur J Heart Fail. (2002) 4:531-9. doi: 10.1016/S1388-9842(02)0 0034-X

36. Deedwania PC, Giles TD, Klibaner M, Ghali JK, Herlitz J, Hildebrandt P, et al. Efficacy, safety and tolerability of metoprolol CR/XL in patients with diabetes and chronic heart failure: experiences from MERIT-HF. Am Heart J. (2005) 149:159-67. doi: 10.1016/j.ahj.2004.05.056

37. Iwaoka M, Obata JE, Abe M, Nakamura T, Kitta Y, Kodama Y, et al. Association of low serum levels of apolipoprotein A-I with adverse outcomes in patients with nonischemic heart failure. J Card Fail. (2007) 13:24753. doi: 10.1016/j.cardfail.2007.01.007

38. Kurano M, Tsukamoto K, Shimizu T, Kassai H, Nakao K, Aiba A, et al Protection against insulin resistance by apolipoprotein $\mathrm{M} /$ sphingosine-1phosphate. Diabetes. (2020) 69:867-81. doi: 10.2337/db19-0811

39. Aboumsallem JP, Mishra M, Amin R, Muthuramu I, Kempen H, De Geest B. Successful treatment of established heart failure in mice with recombinant HDL (Milano). Br J Pharmacol. (2018) 175:4167-82. doi: 10.1111/bph.14463

Conflict of Interest: The authors declare that the research was conducted in the absence of any commercial or financial relationships that could be construed as a potential conflict of interest.

Publisher's Note: All claims expressed in this article are solely those of the authors and do not necessarily represent those of their affiliated organizations, or those of the publisher, the editors and the reviewers. Any product that may be evaluated in this article, or claim that may be made by its manufacturer, is not guaranteed or endorsed by the publisher.

Copyright (c) $2021 \mathrm{Xu}$, Wang, Liu, Meng, Long, Wang and Liu. This is an open-access article distributed under the terms of the Creative Commons Attribution License (CC $B Y)$. The use, distribution or reproduction in other forums is permitted, provided the original author(s) and the copyright owner(s) are credited and that the original publication in this journal is cited, in accordance with accepted academic practice. No use, distribution or reproduction is permitted which does not comply with these terms. 